\title{
Laryngeal injury from a horse bite to the neck
}

\author{
Susan C Lee*, Elizabeth Rossmann Beel, Jaime Ortiz, Frances Karam Cena and Suman Rajagopalan \\ Department of Anesthesiology, Baylor College of Medicine, Houston, Texas, USA
}

\begin{abstract}
Trauma to the neck can present significant challenges to securing an airway. Isolated fractures of the larynx are uncommon injuries. They are frequently associated with difficult mask ventilation and intubation that can result in significant morbidity. We present a case of a 56 years old male with increasing respiratory distress following a horse bite to the neck. An awake fiberoptic intubation was attempted but was unsuccessful. Hence, a decision to quickly proceed with a cricothyroidotomy was made. It is important to immediately recognize the need for a surgical airway to decrease the risk of airway related complications.
\end{abstract}

\section{Case report}

A fifty-six year old male was transferred to our Level One trauma center for increasing respiratory distress following a horse bite to the neck. He presented with labored breathing, drooling with bloody secretions, and hoarseness of voice. Crepitus was noted throughout the anterior and posterior aspect of the neck. The patient was immediately taken to the operating room for establishment of a definitive airway. An awake fiberoptic intubation was attempted with topicalization of the airway with $4 \%$ lidocaine. Visualization of the vocal cords was challenging due to copious amounts of bloody secretions in addition to the distorted airway anatomy. As the patient began to exhibit more respiratory distress and oxygen saturations were in the low 90 s, a decision was quickly made to proceed with a surgical airway. Local anesthesia was administered at the surgical site and an awake cricothyroidotomy was performed by the surgical team with the patient in a semi-recumbent position. A 6.0-cuffed endotracheal tube was successfully placed into the trachea.

A computerized tomography (CT) scan done after the procedure showed comminuted fractures of the thyroid cartilage, significant glottic and supraglottic soft tissue edema, and air throughout the infrahyoid and suprahyoid fascial planes (Figure 1). The next day, he underwent open reduction internal fixation of the thyroid cartilage and a formal tracheostomy. Due to the severity of the mucosal injury, a laryngeal stent was also placed into the endolarynx and sutured
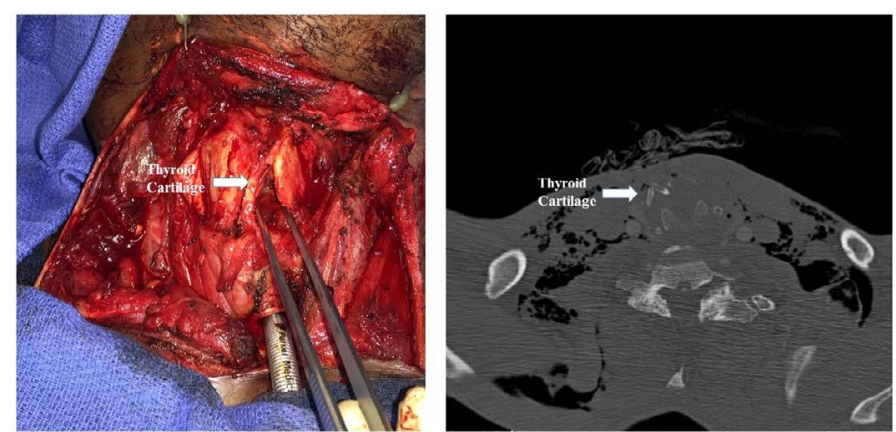

Figure 1: Left panel: photograph of the airway secured by cricothyroidotomy, arrow is pointing to the fractured thyroid cartilage; Right panel: computerized tomography scan of the neck shows the thyroid cartilage broken in four pieces through the thyroid cartilage to silastic buttons on the skin. Laryngeal stents served as internal fixation devices to help prevent endolaryngeal scarring. Indications for stent placement include multiple cartilaginous fractures, anterior commissure lacerations, and significant distortion of laryngeal architecture, all of which were present in this patient.

The patient improved over the course of his admission and the laryngeal stent was removed at three weeks. A flexible fiberoptic laryngoscopic examination at that time showed that the true vocal cords were both mobile and functional. He was discharged home with a tracheostomy.

\section{Discussion}

Trauma to the larynx can be a life-threatening injury and may require immediate surgical intervention. As a part of normal aging, the laryngeal framework consisting of the cricoid and thyroid cartilage becomes increasingly ossified and liable to fracture. The musculotendinous attachment of this framework allows for good lateral force deflection, but application of anterior force to the neck can cause laryngeal compression and fracture. Surgical restoration of the laryngeal framework in a timely fashion can ideally allow for return of full laryngeal function including airway, voice, and deglutition [1]. However, the anatomy and tissues of the laryngeal framework make the immobilization necessary for primary healing challenging [2]. Early intervention has been associated with higher likelihood of return to baseline voice [3]. If fractures are not repaired promptly, laryngeal stenosis, hoarseness / aphonia, and loss of deglutition can occur either acutely or over time as the fracture heals in a misaligned fashion [4].

The severity of laryngeal injuries can be assessed using the SchaeferFuhrman classification, which assigns grades based on amount of mucosal damage and present and extent of fractures. Grades I-II are less severe and may not require surgical intervention [1,5]. Higher

${ }^{*}$ Correspondence to: Susan C Lee, Department of Anesthesiology, Baylor College of Medicine, One Baylor Plaza, Mail Stop BCM120, Houston, Texas 77030, USA, E-mail: scl@bcm.edu

Key words: tracheal injury, horse bite, laryngeal fractures, cricothryoidotomy

Received: April 21, 2018; Accepted: May 17, 2018; Published: May 24, 2018 
grade injuries III-V are more likely to require reconstruction of the laryngeal framework with open reduction and internal fixation. If substantial intraluminal mucosal injury or complete laryngo-tracheal separation is present, stents may also be required. Because laryngeal injuries are relatively rare, even in level 1 trauma centers, few surgeons have the necessary experience to repair these types of injuries. No specific guidelines or protocols currently exist for the management of traumatic laryngeal injuries [1].

The traditional approach to laryngeal injury has been primary repair with sutures to approximate soft tissue defects combined with wires for stabilization of cartilage. However, laryngeal motion during swallowing, speaking, and neck movement leads to bending of these wires with subsequent angulation and loss of reduction of the fracture [4]. More recently, mini-plates with screws allow for more rigid and threedimensional fixation and can also be used to fix larger defects. Because mini-plates can better approximate and stabilize the original laryngeal dimensions, late stenotic complications are thought to be reduced [2].
In conclusion, isolated fractures of the larynx are uncommon injuries. They are frequently associated with difficult mask ventilation and intubation that can result in significant morbidity. Hence, when there is a high suspicion for laryngeal/tracheal injury, a surgical airway should be considered early in the airway management.

\section{References}

1. Jalisi S, Zoccoli M (2011) Management of laryngeal fractures: A 10-year experience. $J$ Voice 25: 473-479. [Crossref]

2. Woo P (1990) Laryngeal framework reconstruction with miniplates. Ann Otol Rhinol Laryngol 99: 772-777. [Crossref]

3. Juutilainen M, Vintturi J, Robinson S, Bäck L, Lehtonen H, et al. (2008) Laryngeal fractures: clinical findings and considerations on suboptimal outcome. Acta Otolaryngol 128: 213-218. [Crossref]

4. De Mello-Filho FV, Carrau RL (2000) The management of laryngeal fractures using internal fixation. The Laryngoscope 110: 2143-2146. [Crossref]

5. Fuhrman GM, Stieg, FH, Buerk CA (1990) Blunt laryngeal trauma: classification and management protocal. J Trauma 30: 87-92. [Crossref]

Copyright: C2018 Lee SC. This is an open-access article distributed under the terms of the Creative Commons Attribution License, which permits unrestricted use, distribution, and reproduction in any medium, provided the original author and source are credited. 